\title{
miR-135b inhibits tumour metastasis in prostate cancer by targeting STAT6
}

\author{
NING WANG ${ }^{1 *}$, LIANGJUN TAO ${ }^{2 *}$, HUAN ZHONG $^{1}$, SIHAI ZHAO $^{1}$, YING YU $^{1}$, \\ BIN YU $^{1}$, XIAONONG CHEN ${ }^{1}$, JIANGUO GAO ${ }^{1}$ and RONGJIANG WANG ${ }^{1}$ \\ ${ }^{1}$ Department of Urology, The First Affiliated Hospital of Huzhou Teachers College, Huzhou, Zhejiang 313000; \\ ${ }^{2}$ Department of Urology and Institute of Prostatic Diseases, \\ The Affiliated Wuhu No. 2 People's Hospital of Wannan Medical College, Wuhu, Anhui 241000, P.R. China
}

Received December 1, 2014; Accepted October 5, 2015

DOI: $10.3892 / \mathrm{ol} .2015 .3970$

\begin{abstract}
MicroRNAs (miRNAs) are small non-coding RNAs that participate in several cellular functions and tumour progression. A previous microarray study demonstrated that miR-135b is downregulated in prostate cancer ( $\mathrm{PCa}$ ) cells, but the role and molecular mechanism of miR-135b in the regulation of tumour metastasis remain to be elucidated. In the present study, significant downregulation of miR-135b in $\mathrm{PCa}$ tissues, compared with noncancerous tissues, was detected by reverse transcription-quantitative polymerase chain reaction. Furthermore, the expression of miR-135b was demonstrated to be associated with the pathological stage and the levels of total and free prostate-specific antigen (PSA) in PCa cells. In addition, signal transducer and activator of transcription 6 (STAT6) was identified as a target of miR-135b in PCa cells by luciferase activity and western blot assays. The upregulation of miR-135b in PCa cells led to reduced expression of STAT6 in the cytoplasm and nucleus of these cells, while the overexpression of miR-135b and knockdown of STAT6 were able to inhibit the migration and invasion abilities of PCa cells in vitro. Therefore, the results of the present study indicate that miR-135b suppresses tumour metastasis by targeting STAT6.
\end{abstract}

\section{Introduction}

Prostate cancer ( $\mathrm{PCa}$ ) is the most common malignancy affecting men worldwide, and the second leading cause of cancer-associated mortalities in western countries (1). The complexity of $\mathrm{PCa}$ is due to it diverse metastasis profile, heterogeneous degree of aggressiveness and variable response

Correspondence to: Professor Rongjiang Wang, Department of Urology, The First Affiliated Hospital of Huzhou Teachers College, 158 Guangchanghou Road, Huzhou, Zhejiang 313000, P.R. China E-mail:wn6530289162@163.com; drtaoliangjun@126.com

*Contributed equally

Key words: miR-135b, metastasis, prostate cancer, STAT6 to conventional therapies (2). Although PCa may be controlled temporarily by hormone deprivation, it eventually becomes refractory to hormonal therapy, and no effective treatment has been developed thus far for this type of hormone-insensitive cancer (3). Therefore, the development of novel drugs for the treatment of $\mathrm{PCa}$, which offer higher specificity and potentially improved prognosis and clinical outcomes for patients with $\mathrm{PCa}$, is required (4).

MicroRNAs (miRNAs) are a class of single-stranded small non-coding RNAs, of 17-27 nucleotides in length, which negatively regulate the expression of target genes by binding to the 3'-untranslated regions (UTRs) of their messenger (m) RNAs, thus inhibiting the process of protein translation or promoting the degradation of these mRNAs (5). By regulating the expression of genes associated with cancer, miRNAs may act as oncogenes or tumour suppressors $(6,7)$. Recent studies have reported the dysregulation of miRNAs in human tumours, which suggests that miRNAs participate in the pathogenesis of cancer, including disease onset, progression and metastasis $(8,9)$.

miR-135b has been previously associated with human colorectal (10) and breast cancer (11), and its expression appears to be downregulated in PCa cells (12). However, functional analysis of miR-135b in PCa has not been conducted thus far. In the present study, in silico analysis of 3'-UTRs identified signal transducer and activator of transcription 6 (STAT6) as a putative target of miR-135b. Activated STAT6 induces the expression of various genes involved in cell differentiation, proliferation, metastasis and resistance to apoptosis (13-15). It has been previously observed that STAT6 is overexpressed and activated in numerous malignancies, including PCa (16), colon cancer (17), lymphoma (18-20) and leukemia $(21,22)$. In addition, STAT6 was identified as a robust marker gene for human PCa in previous DNA microarray studies (23).

Therefore, the aim of the present study was to investigate the association between miR-135b and its potential target STAT6 in PCa cells.

\section{Materials and methods}

Cell culture and tissue collection. PCa cell lines DU145 and PC3 were purchased from the Shanghai Cell Bank, Chinese Academy of Sciences (Shanghai, China), and maintained 
in Dulbecco's modified Eagle's medium (DMEM) (Gibco, Thermo Fisher Scientific, Inc., Waltham, MA, USA) supplemented with $10 \%$ fetal bovine serum (FBS; GE Healthcare Life Sciences, Logan, UT, USA), $100 \mathrm{U} / \mathrm{ml}$ penicillin and $100 \mu \mathrm{g} / \mathrm{ml}$ streptomycin (KeyGen Biotech. Co. Ltd., Nanjing, China), in an atmosphere of $5 \% \mathrm{CO}_{2}$ and $37^{\circ} \mathrm{C}$. PCa and nonmalignant prostate tissues were collected from patients who underwent radical prostatectomy at the Department of Urology of The First Affiliated Hospital of Huzhou Teachers College (Huzhou, China). The use of clinical specimens in the present study was approved by the medical ethics committee of The First Affiliated Hospital of Huzhou Teachers College. Detailed information of each tissue donor is provided in Table I.

Cell transfection. Homo sapiens (Hsa)-miRNA-135b and small interfering (si)RNAs S-1 and S-2 were chemically synthesized by Gene Pharma Co., Ltd. (Shanghai, China). The sequence of Hsa-miRNA-135b mimics were as follows: Sense, 5'-UAUGGCUUUUCAUUCCUAUGUGA-3', and anti-sense, 5'-ACAUAGGAAUGAAAGCCAUAUU-3'. The sequence of the negative controls were as follows: Sense, 5'-UUCUCC GAACGUGUCACGUTT-3', and anti-sense, 5'-ACGUGACAC GUUCGGAGAATT-3'. The sequences of siRNA-STAT6 S-1 were, 5'-AAGCAGGAAGAACUCAAGUUUTT-3' (sense) and 5'-AAACUUGAGUUCUUCCUGCUUTT-3' (anti-sense), while for siRNA-STAT6 S-2 the sequences were 5'-AAA CGAGAGUGUUAUAACUGUTT-3' (sense) and 5'-ACAGUU AUAACACUCUCGUUUTT-3' (anti-sense). Cells at 50-60\% confluence were transfected with the corresponding oligonucleotide using Lipofectamine 2000 (Invitrogen, Thermo Fisher Scientific, Inc.), according to the manufacturer's protocol.

Reverse transcription-quantitative polymerase chain reaction (RT-qPCR). Total RNA was extracted using TRIzol (Invitrogen, Thermo Fisher Scientific, Inc.). For amplification of miR-135b, RT-qPCR was performed with TaqMan MicroRNA Assays (Applied Biosystems, Thermo Fisher Scientific, Inc.), using U6 as internal control. The reaction conditions were as follows: 40 cycles of $95^{\circ} \mathrm{C}$ for $10 \mathrm{~min}, 95^{\circ} \mathrm{C}$ for $15 \mathrm{sec}$ and $60^{\circ} \mathrm{C}$ for $1 \mathrm{~min}$. Relative expression levels were calculated by the $2^{-\Delta \Delta C q}$ method.

Immunofluorescence analysis. Transfected DU145 cells were seeded on coverslips, stimulated with $50 \mathrm{ng} / \mathrm{ml}$ human interleukin (IL)-4 (GE Healthcare Life Sciences) for 30 min, fixed with $4 \%$ paraformaldehyde (KeyGen Biotech. Co. Ltd.), blocked with $3 \%$ bovine serum albumin (BSA; GE Healthcare Life Sciences) for $1 \mathrm{~h}$, and incubated with primary antibodies overnight at $4^{\circ} \mathrm{C}$. Fluorescein isothiocyanate-labeled secondary antibody was then added for $2 \mathrm{~h}$ at $37^{\circ} \mathrm{C}$, and following staining with DAPI (KeyGen Biotech. Co. Ltd.) for $5 \mathrm{~min}$, the cells were subjected to confocal laser scanning (Nikon Corporation, Tokyo, Japan).

Migration and invasion assays. At $24 \mathrm{~h}$ post-transfection, PC3 and DU145 cells were added to the upper chamber of a 24-well plate with $8-\mu \mathrm{m}$ pores (BD Biosciences, San Jose, CA, USA), which was coated with Matrigel (BD Biosciences) for invasion assays, and $0.7 \mathrm{ml}$ of $20 \%$ FBS-DMEM was then added to the
Table I. Clinical characteristics of the 32 patients with prostate cancer who participated in the present study.

\begin{tabular}{lc}
\hline Characteristics & No. of patients \\
\hline Age (n=32) & \\
Median (range) & $70(56-76)$ \\
T stage ( $\mathrm{n}=32)$ & \\
T1 & 2 \\
T2 & 17 \\
T3 & 12 \\
T4 & 1 \\
N stage & \\
N0 & 27 \\
N1 & 5 \\
M stage & \\
M0 & 0 \\
M1 & 32 \\
Total PSA at diagnosis $(\mathrm{n}=29)$ & \\
Median (range) & \\
Free PSA at diagnosis $(\mathrm{n}=26)$ & \\
Median (range) & \\
Gleason score (n=32) & \\
$<7$ & 18.00 \\
$=7$ & 10 \\
$>7$ & 10 \\
\hline
\end{tabular}

PSA, prostate-specific antigen.

lower chamber. Following incubation for $48 \mathrm{~h}$, non-migrated or non-invaded cells were removed from the upper well by cotton swabs, while the migrated or invaded cells were fixed with $95 \%$ methanol, stained with $0.1 \%$ crystal violet (KeyGen Biotech. Co. Ltd.), and photographed in five independent fields/well.

Luciferase reporter assay. For the luciferase reporter assay, cells were cotransfected with wild-type or mutant STAT6 3'-UTR reporter plasmid and miR-135b, using Lipofectamine 2000. For normalization, 1 ng pRLSV40 Renilla reniformis luciferase construct (Promega Corporation, Madison, WI, USA) was used. Luciferase assays were performed at $48 \mathrm{~h}$ post-transfection, using Dual-Luciferase Reporter Assay System (Promega Corporation).

Western blot analysis. Total protein was collected with Total Protein Extraction Kit (KeyGen Biotech. Co. Ltd., Nanjing, China). Proteins $(30 \mathrm{mg})$ were separated on a $10 \%$ sodium dodecyl sulfate-polyacrylamide gel, and transferred electrophoretically onto polyvinylidene difluoride membranes (BD Biosciences). The membranes were blocked in 5\% skimmed milk for $1 \mathrm{~h}$, and then incubated for $2 \mathrm{~h}$ with rabbit anti-STAT6 (1:1,000; Abcam, Cambridge, MA, USA), rabbit anti-phosphorylated (p)-STAT6 (1:1,000; Cell Signaling Technology, Inc., Danvers, MA, USA) or rabbit anti-GAPDH (1:1,000; Bioworld, Technology, Inc., St. Louis Park, MN, USA), which 
was used as loading control. Following incubation with goat anti-rabbit secondary antibody (Bioworld, Technology, Inc.), the signals derived from the antibody-antigen binding reaction were detected by enhanced chemiluminescence (BD Biosciences).

Statistical analysis. Data are reported as the mean \pm standard error of the mean. All experiments were conducted in triplicate. The correlation between downregulation of miR-135b and levels of total or free PSA in the PCa samples was analysed using the Spearman rank correlation test. All other data were compared by two-sided t-test. Statistical analysis was performed with SPSS software version 17.0 (SPSS, Inc., Chicago, IL, USA). P<0.05 was considered to indicate a statistically significant difference.

\section{Results}

miR-135b is significantly downregulated in PCa tissues. The expression of miR-135b was analyzed in 32 primary $\mathrm{PCa}$ samples and 14 nonmalignant samples by RT-qPCR. The results revealed that the expression of miR-135b was significantly downregulated in $\mathrm{PCa}$ tissues, compared with noncancerous tissues (Fig. 1A). The results also indicated that the expression levels of miR-135b were possibly correlated with the pathological T stages of PCa (Fig. 1A). Furthermore, the results demonstrated significant inverse correlations between the expression levels of miR-135b and the levels of total and free PSA (Fig. 1B and C, respectively). No statistical significant difference was observed for the association between the expression levels of miR-135b and the Gleason scores (Fig. 1D).

miR-135b inhibited PCa cell migration and invasion. The role of miR-135b on cell migration and invasion, two key determinants of malignant progression and metastasis, was assessed in human PCa cells. A significant decrease in cell migration was observed in miR-135b-mimics-transfected PC3 and DU145 cells, compared with negative control (NC)-transfected cells (Fig. 2A). The effect of miR-135b on cell invasion across the extracellular matrix was evaluated, and it was observed that the ectopic expression of miR-135b also reduced the invasion ability of PCa cells (Fig. 2B). These results suggested a mechanism by which the overexpression of miR-135b may contribute to the inhibition of tumour progression and metastasis in human $\mathrm{PCa}$.

STAT6 was targeted by miR-135b. To explore the mechanism by which miR-135b regulates cell metastasis, a miRNA target search was performed with three target prediction programs, including TargetScan (http://www.targetscan.org/), miRanda (http://www.microrna.org/microrna/home.do) and PicTar (http://pictar.mdc-berlin.de/), and highly conserved putative binding sites for miR-135b were identified in the 3'-UTR of STAT6 (Fig. 3A). When miR-135b-mimics were cotransfected with the reporter plasmids, the relative luciferase activity of the reporter plasmid containing wild-type STAT6 3'-UTR was markedly reduced, while the luciferase activity of the reporter plasmid containing mutant STAT6 3'-UTR was unaltered (Fig. 3D). Furthermore, western blotting analysis was conducted to determine whether the expression of STAT6 was regulated by miR-135b. As presented in Fig. 3B, the protein expression
A

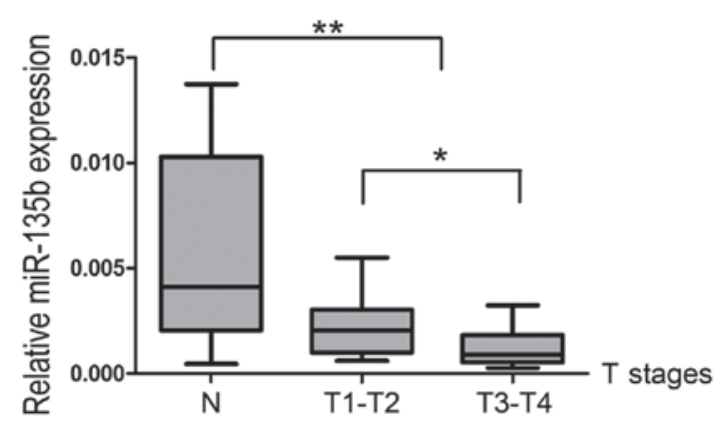

B

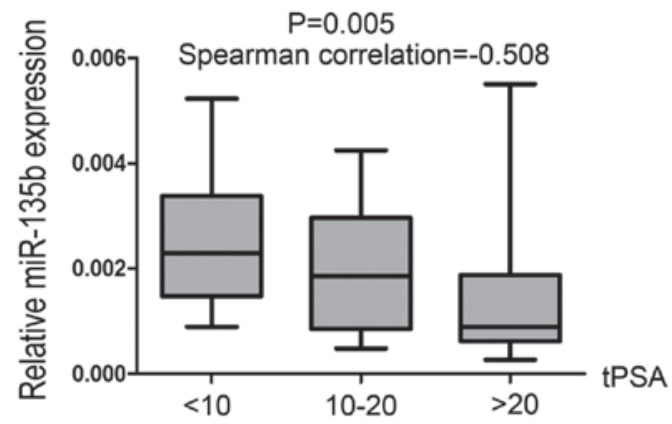

C

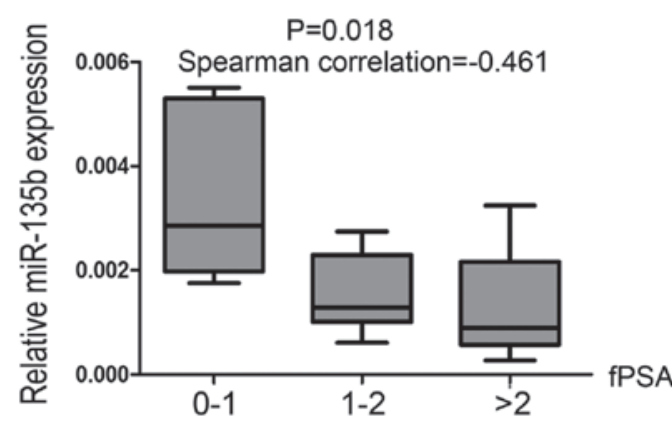

D

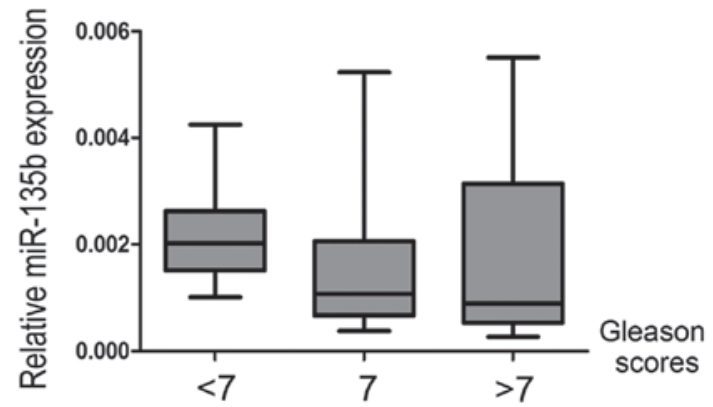

Figure 1. Downregulation of miR-135b is associated with clinicopathological features of PCa. (A) The expression levels of miR-135b in noncancerous tissues and in PCa tissues at pathological stages T1-T4 were analysed by reverse transcription-quantitative polymerase chain reaction. Data are presented as the mean \pm SEM. ${ }^{*} \mathrm{P}<0.05$ and ${ }^{* *} \mathrm{P}<0.01$ by t-test. A statistically significant inverse correlation was observed between the expression levels of miR-135b and the levels of (B) total and (C) free prostate-specific antigen (Spearman correlation $=-0.508, \mathrm{P}=0.005$; and Spearman correlation $=-0.461, \mathrm{P}=0.018$, respectively). (D) Association between the expression levels of miR-135b and the Gleason scores. Data are presented as the mean \pm SEM. $\mathrm{P}>0.05$ by t-test. miR, microRNA; PCa, prostate cancer; SEM, standard error of the mean; N, noncancerous; tPSA, total prostate-specific antigen; fPSA, free prostate-specific antigen. 
A
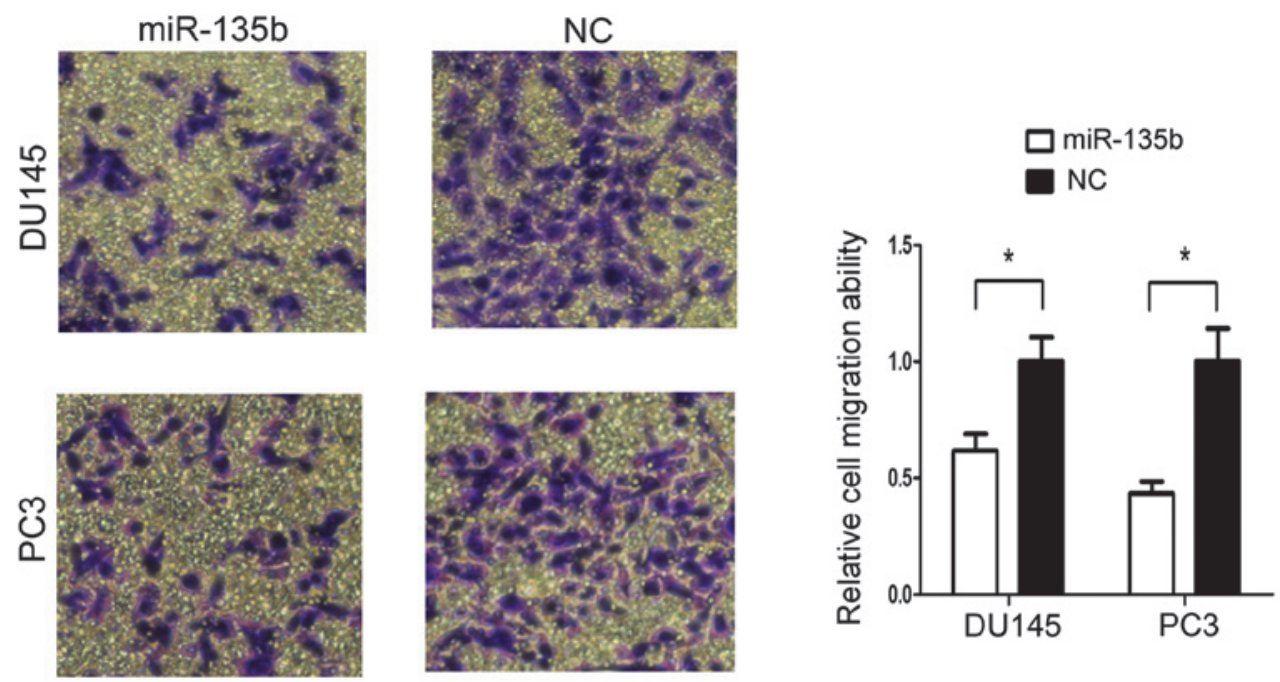

B
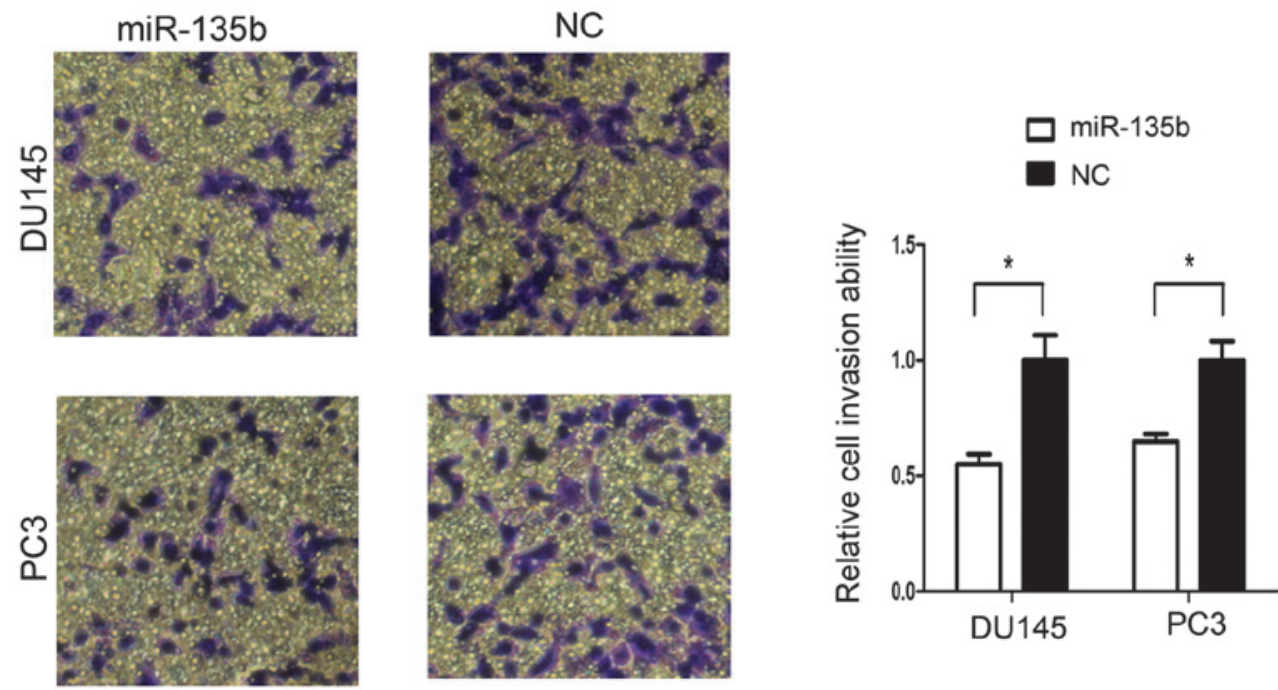

Figure 2. Overexpression of miR-135b in PCa cells inhibits cell migration and invasion in vitro. Upregulation of miR-135b inhibited cell (A) migration and (B) invasion in the PCa cell lines PC3 and DU145. Data are presented as the mean \pm standard error of the mean of $\leq 3$ independent experiments (magnification, x200). ${ }^{*} \mathrm{P}<0.05$ by t-test. miR, microRNA; PCa, prostate cancer; NC, negative control.

levels of STAT6 and p-STAT6 were markedly downregulated following transfection with miR-135b mimics. These results indicated that miR-135b may downregulate the expression of STAT6 through the miR-135-binding sequences located at the 3'-UTR of the STAT6 gene.

miR-135b reduced the IL-4-induced nuclear translocation of STAT6. The effect of miR-135b on the cellular localization of STAT6 was examined by immunofluorescence analysis. Under basal conditions, the immunofluorescent signal corresponding to STAT6 was located predominantly in the cytoplasm of PC3 cells (Fig. 3C). The levels of STAT6 were reduced in the cytoplasm of $\mathrm{PC} 3$ cells following transfection with miR-135b mimics (Fig. 3C). Treatment of DU145 cells with IL-4 $(50 \mathrm{ng} / \mathrm{ml})$ for $30 \mathrm{~min}$ resulted in marked translocation of STAT6 to the nucleus (Fig. 3C). Furthermore, the IL-4-induced nuclear translocation of STAT6 in DU145 cells was reduced following transfection with miR-135b mimics (Fig. 3C).
Knockdown of STAT6 mimicked miR-135b inhibition. To investigate if miR-135b mediates its metastasis-suppressive effects primarily through knocking down STAT6, PC3 and DU145 cells were transfected with two siRNAs-STAT6 (S-1 and S-2). The results of western blot analysis indicated that $\mathrm{S}-1$ and $\mathrm{S}-2$ knocked down the expression of STAT6 at the protein level (Fig. 4A). In addition, the effect of siRNA-STAT6 S-1 on the migration and invasion abilities of PCa cells was analysed in subsequent experiments. As expected, compared with NC-transfected cells, STAT6-knockdown DU145 and PC3 cells exhibited reduced migration and invasion abilities (Fig. 4B and C), similarly to the phenotype displayed upon miR-135b restoration.

\section{Discussion}

Previous studies have reported that miR-135b is overexpressed in embryonic stem cells and colorectal cancer $(24,25)$, and a previous microarray profiling of human head and neck 
A

Hsa-miR-135b

Position 1100-1107 of STAT6 3'-UTR

Mutant STAT6 3'-UTR

5... GGGGUCACAGAUCCL-AAGUUAUA

C
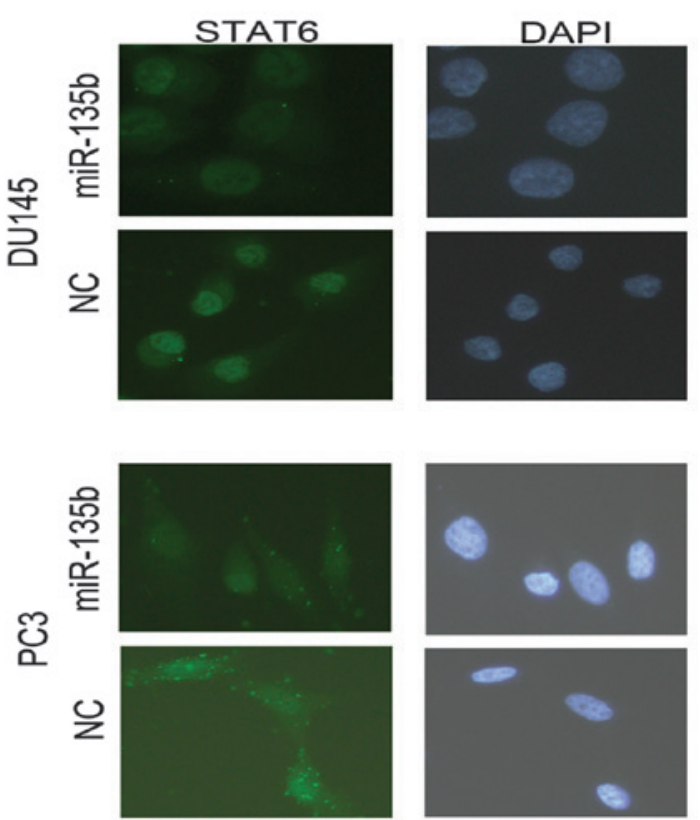

B
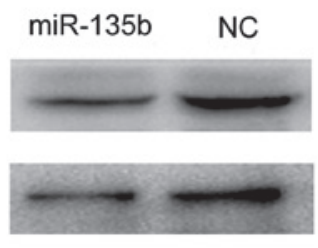

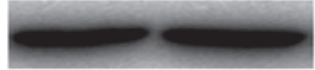

DU145
STAT6

p-STAT6
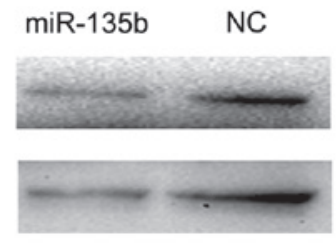

GAPDH

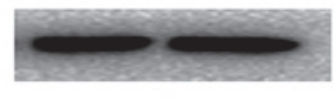

PC3
D

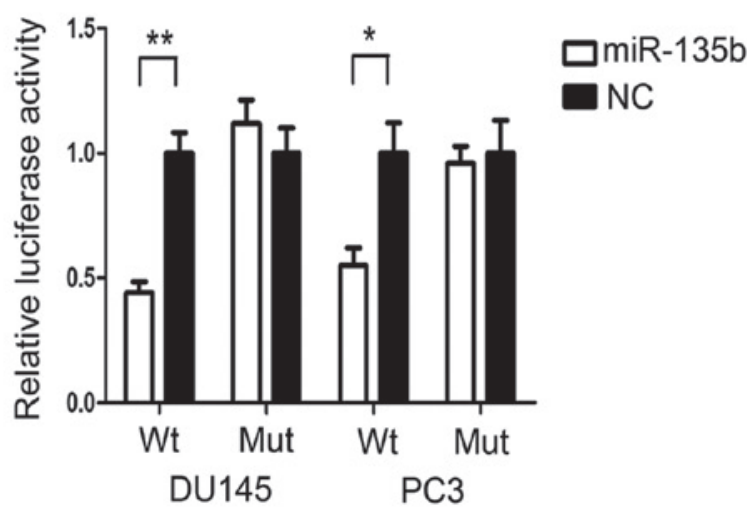

Figure 3. STAT6 is a direct target gene of miR-135b. (A) Schematic description of the 3'-untranslated region of STAT6 with two putative binding sites for miR-135b. (B) The protein levels of STAT6 and phosphorylated-STAT6 were reduced in the human PCa cell lines DU145 and PC3 following transfection with miR-135b. (C) Upregulation of miR-135b in PCa cells led to reduced expression of STAT6 in the cytoplasm and nucleus of these cells. (D) Luciferase reporter assay was performed in DU145 and PC3 cells and indicated that miR-135b targets STAT6 through the miR-135-binding sequences located at the 3'-UTR of the STAT6 gene. Data are presented as the mean \pm standard error of the mean. ${ }^{*} \mathrm{P}<0.05$ and ${ }^{* *} \mathrm{P}<0.01$ by t-test. STAT6, signal transducer and activator of transcription 6; miR, microRNA; p, phosphorylated; PCa, prostate cancer; Hsa, Homo sapiens; UTR, untranslated region; NC, negative control; GAPDH, glyceraldehyde 3-phosphate dehydrogenase; DAPI, 4',6-diamidino-2-phenylindole; Wt, wild-type; Mut, mutant.

squamous cell carcinoma samples demonstrated that miR-135b was one of the most significantly upregulated miRNAs in this type of cancer (26). The expression of miR-135b in $\mathrm{PCa}$ is controversial, since miR-135b was previously observed to be upregulated in certain PCa tissues (27) but downregulated in certain PCa cell lines (28). However, no functional evidence for a role of miR-135b as an oncogenic or tumour-suppressive miRNA has been documented thus far. In the present study, miR-135b appeared to be markedly downregulated in $\mathrm{PCa}$ tissues, compared with normal tissues. Previous clinical observations have suggested that miR-135b may be downregulated in oral squamous cell carcinoma, particularly in the advanced stage (29), which is consistent with the results of the present study. Furthermore, the expression levels of miR-135b were observed to be inversely correlated with the levels of total and free PSA. Previous studies have suggested that miR-135b may be a potential diagnostic and prognostic marker for colorectal cancer (30-32). These results indicate that, as a tumour-suppressor gene, miR-135b may be used as a marker for early diagnosis of $\mathrm{PCa}$ and for detecting tumour progression. Due to the limited number of samples in the present study, it is not possible to confirm whether the expression of miR-135b is associated with the Gleason scores. Therefore, further studies with a larger number of clinical samples are required.
Since the majority of cancer-associated mortalities are caused by dissemination of the disease rather than the primary tumour, metastasis has become the most prominent problem in the clinical treatment of cancer (33). There is increasing evidence suggesting that miRNAs participate in tumour metastasis (34), since miRNAs may influence multiple steps of the metastatic cascade, including cell migration, invasion and intravasation. A previous study has revealed that dysregulation of miR-221 and miR-222 was associated with cancer progression, poor prognosis and development of metastases in patients with PCa (35). It has been previously demonstrated that miR-146a targets Rho-associated coiled-coil containing protein kinase 1 (ROCK1), and that high levels of ROCK1 promote cell proliferation, invasion and metastasis in $\mathrm{PCa}$ cells (36). The results of the gain-of-function experiments conducted in the present study indicated that miR-135b was positively correlated with the invasive and migratory abilities of PCa cells in vitro, since upregulation of miR-135b in the human PCa cell lines DU145 and PC3, which normally exhibit high metastatic ability and low expression levels of miR-135b, reduced the metastatic ability of these cells.

The STAT family of transcription factors consists of seven members (STAT 1-4, 5a, 5b and 6), which can be activated by a number of cytokines, hormones and growth factors (37). Upon activation, STATs dissociate from their 
A

\section{DU145}

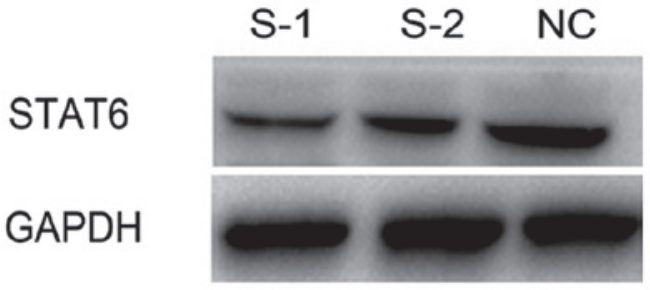

B
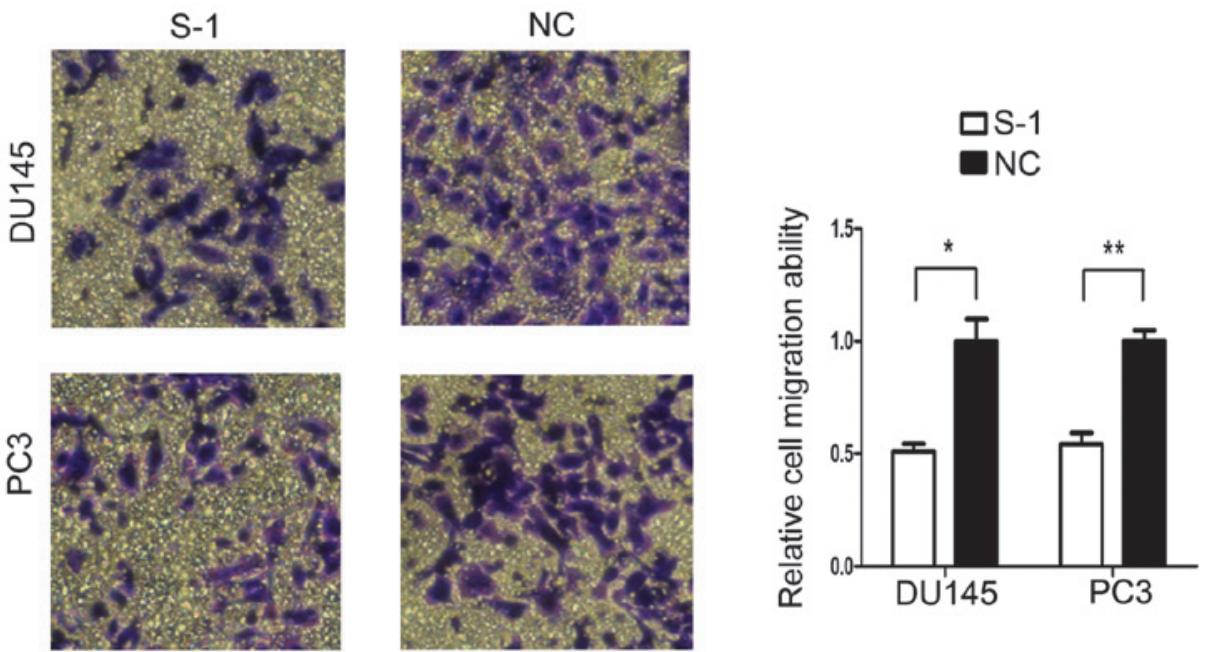

C
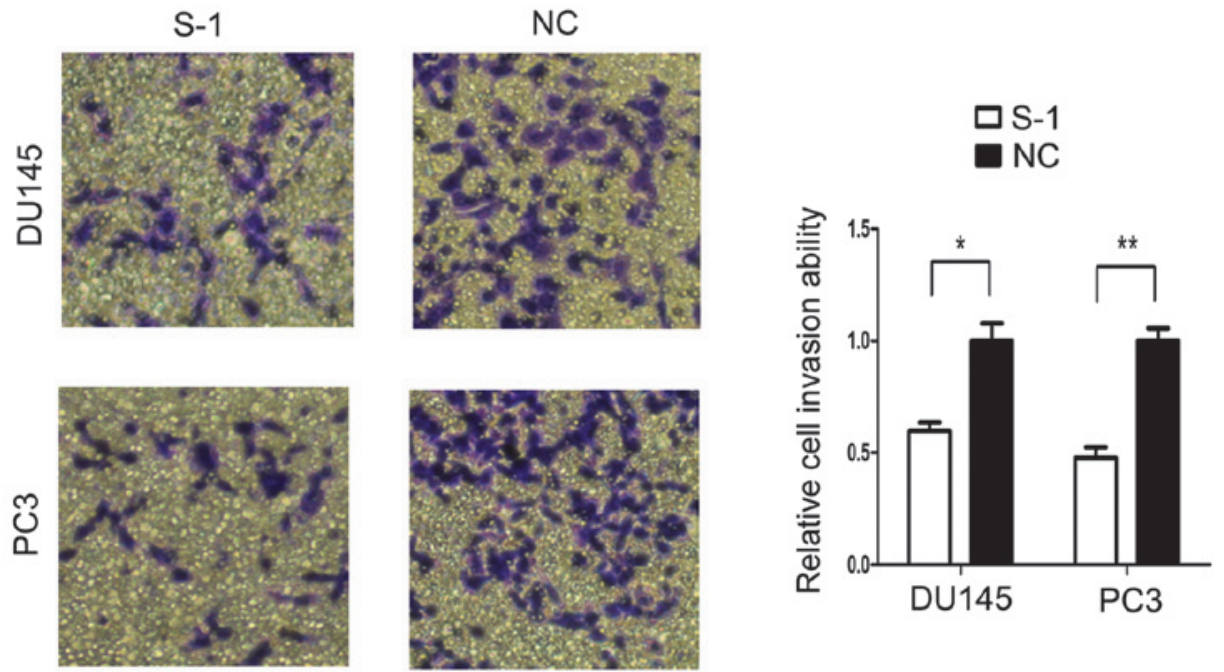

Figure 4. Effects of STAT6 knockdown on the cell migration and invasion abilities of the human prostate cancer cell lines DU145 and PC3. (A) The protein expression levels of STAT6 were assessed by western blotting in DU145 cells transfected with two small interfering RNAs-STAT6 (S-1 and S-2), and compared with those in DU145 cells transfected with a negative control. Knocking down STAT6 led to reduced (B) migratory and (C) invasive capacity in DU145 and PC 3 cells. Data are presented as the mean \pm standard error of the mean. " $\mathrm{P}<0.05$ and ${ }^{* *} \mathrm{P}<0.01$ by $\mathrm{t}$-test. STAT6, signal transducer and activator of transcription 6; NC, negative control; GAPDH, glyceraldehyde 3-phosphate dehydrogenase.

receptor, form dimmers, translocate to the nucleus and bind to cognate DNA response elements, thus activating the transcription of their target genes $(38,39)$. STAT6 is activated through tyrosine phosphorylation by the cytokines IL-4 and -13 (22). It has been previously reported that the activity of STAT6 in PCa tissues and cell lines is significantly higher than in normal tissues and cell lines (40). In the present study, STAT6 was confirmed to be a target of miR-135b in PCa cells by luciferase reporter assay, and overexpression of miR-135b in these cells was observed to reduce the expression levels of STAT6 by western blot assay. Previous immunofluorescence studies have reported that STAT6 is located predominantly in the cytoplasm of corneal fibroblasts, and IL-4 markedly induced the translocation of STAT6 to the nucleus of these cells (41). These findings are consistent with the results of the present study, whereby the expression levels of STAT6 in the cytoplasm of 
the PCa cell lines tested, and the IL-4-induced levels of STAT6 in the nucleus of these cells, were observed to be reduced upon transfection with miR-135b mimics. These results indicated that the upregulation of miR-135b inhibited the expression of STAT6 and reduced the IL-4-induced nuclear translocation of STAT6 in PCa cells. Previous studies have reported that mice lacking STAT6 exhibited higher tumour immunity to primary and metastatic mammary carcinoma, compared with normal mice $(42,43)$. In the present study, knocking down STAT6 significantly reduced the metastatic potential of $\mathrm{PCa}$ cells in vitro. This is consistent with the aforementioned findings regarding the correlation between the upregulation of miR-135b in PCa cells and the reduced aggressive phenotype displayed by these cell lines. Taken together, the data of the present study indicate that miR-135b modulates the metastatic ability of PCa cells by regulating the expression of STAT6.

In conclusion, the findings of the present study suggest that miR-135b functions as a tumour suppressor, affecting the metastatic ability of PCa cells by targeting STAT6, since STAT6 knockdown resulted in reduced cell metastasis. In addition, miR-135b was able to reduce the IL-4-induced nuclear translocation of STAT6 in these cells. The findings of the present study suggest that miR-135b functions as a tumour suppressor, reducing the metastatic ability of PCa cells by targeting STAT6. Furthermore, the expression of miR-135b was observed to be associated with the pathological $\mathrm{T}$ stages and levels of total and free PSA in patients with PCa. The present study indicates that miR-135b may offer an attractive novel target for diagnostic and therapeutic intervention in PCa.

\section{References}

1. Pflug BR, Pecher SM, Brink AW, Nelson JB and Foster BA: Increased fatty acid synthase expression and activity during progression of prostate cancer in the TRAMP model. Prostate 57: 245-254, 2003.

2. Klein EA, Cooperberg MR, Magi-Galluzzi C, Simko JP, Falzarano SM, Maddala T, Chan JM, Li J, Cowan JE, Tsiatis AC, Cherbavaz D5, et al: A 17-gene assay to predict prostate cancer aggressiveness in the context of Gleason grade heterogeneity, tumor multifocality, and biopsy undersampling. Eur Urol 66: 550-560, 2014

3. FeldmanBJ andFeldmanD:Thedevelopmentof androgen-independent prostate cancer. Nat Rev Cancer 1: 34-45, 2001.

4. Best CJ, Gillespie JW, Yi Y, Chandramouli GV, Perlmutter MA, Gathright Y, Erickson HS, Georgevich L, Tangrea MA, Duray PH, et al: Molecular alterations in primary prostate cancer after androgen ablation therapy. Clin Cancer Res 11: 6823-6834, 2005.

5. Valencia-Sanchez MA, Liu J, Hannon GJ and Parker R: Control of translation and mRNA degradation by miRNAs and siRNAs. Genes Dev 20: 515-524, 2006.

6. Esquela-Kerscher A and Slack FJ: Oncomirs-microRNAs with a role in cancer. Nat Rev Cancer 6: 259-269, 2006.

7. Ventura A and Jacks T: MicroRNAs and cancer: Short RNAs go a long way. Cell 136: 586-591, 2009.

8. Iorio MV and Croce CM: MicroRNAs in cancer: Small molecules with a huge impact. J Clin Oncol 27: 5848-5856, 2009.

9. Baranwal S and Alahari SK: miRNA control of tumour cell invasion and metastasis. Int J Cancer 126: 1283-1290, 2010.

10. Bandrés E, Cubedo E, Agirre X, Malumbres R, Zárate R, Ramirez N, Abajo A, Navarro A, Moreno I, Monzó M and García-Foncillas J: Identification by Real-time PCR of 13 mature microRNAs differentially expressed in colorectal cancer and non-tumoural tissues. Mol Cancer 5: 29, 2006.

11. Lowery AJ, Miller N, Devaney A, McNeill RE, Davoren PA, Lemetre C, Benes V, Schmidt S, Blake J, Ball G and Kerin MJ: MicroRNA signatures predict oestrogen receptor, progesterone receptor and HER2/neu receptor status in breast cancer. Breast Cancer Res 11: R27, 2009.
12. Bhattacharya A, Ziebarth JD and Cui Y: Systematic analysis of microRNA targeting impacted by small insertions and deletions in human genome. PLoS One 7: e46176, 2012.

13. Hebenstreit D, Wirnsberger G, Horejs-Hoeck J and Duschl A: Signaling mechanisms, interaction partners and target genes of STAT6. Cytokine Growth Factor Rev 17: 173-188, 2006

14. Rothstein TL: Inducible resistance to Fas-mediated apoptosis in B cells. Cell Res 10: 245-266, 2000.

15. Ansel KM, Djuretic I, Tanasa B and Rao A: Regulation of Th2 differentiation and I14 locus accessibility. Annu Rev Immunol 24 607-656, 2006.

16. Ni Z, Lou W, Lee SO, Dhir R, DeMiguel F, Grandis JR and Gao AC: Selective activation of members of the signal transducers and activators of transcription family in prostate carcinoma. J Urol 167: 1859-1862, 2002.

17. Li BH, Yang XZ, Li PD, Yuan Q, Liu XH, Yuan J and Zhang WJ: IL-4/Stat6 activities correlate with apoptosis and metastasis in colon cancer cells. Biochem Biophys Res Commun 369: 554-560, 2008.

18. Benekli M, Baer MR, Baumann H and Wetzler M: Signal transducer and activator of transcription proteins in leukemias. Blood 101: 2940-2954, 2003.

19. Skinnider BF,Elia AJ, Gascoyne RD, Patterson B, TrumperL, Kapp U and Mak TW: Signal transducer and activator of transcription 6 is frequently activated in Hodgkin and Reed-Sternberg cells of Hodgkin lymphoma. Blood 99: 618-626, 2002.

20. Guiter C, Dusanter-Fourt I, Copie-Bergman C, Boulland ML, Le Gouvello S, Gaulard P, Leroy K and Castellano F: Constitutive STAT6 activation in primary mediastinal large B-cell lymphoma. Blood 104: 543-549, 2004.

21. Melzner I, Bucur AJ, Brüderlein S, Dorsch K, Hasel C, Barth TF, Leithäuser F and Möller P: Biallelic mutation of SOCS-1 impairs JAK2 degradation and sustains phospho-JAK2 action in the MedB-1 mediastinal lymphoma line. Blood 105: 2535-2542, 2005.

22. Bruns HA and Kaplan MH: The role of constitutively active Stat6 in leukemia and lymphoma. Crit Rev Oncol Hematol 57: 245-253, 2006.

23. Xu L, Tan AC, Naiman DQ, Geman D and Winslow RL: Robust prostate cancer marker genes emerge from direct integration of inter-study microarray data. Bioinformatics 21: 3905-3911, 2005.

24. Lin CH, Jackson AL, Guo J, Linsley PS and Eisenman RN: Myc-regulated microRNAs attenuate embryonic stem cell differentiation. EMBO J 28: 3157-3170, 2009.

25. Nagel $R$, le Sage C, Diosdado B, van der Waal $M$, Oude Vrielink JA, Bolijn A, Meijer GA and Agami R: Regulation of the adenomatous polyposis coli gene by the miR-135 family in colorectal cancer. Cancer Res 68: 5795-5802, 2008.

26. Liu CJ, Tsai MM, Hung PS, Kao SY, Liu TY, Wu KJ, Chiou SH, Lin SC and Chang KW: miR-31 ablates expression of the HIF regulatory factor FIH to activate the HIF pathway in head and neck carcinoma. Cancer Res 70: 1635-1644, 2010.

27. Tong AW, Fulgham P, Jay C, Chen P, Khalil I, Liu S, Senzer N, Eklund AC, Han J and Nemunaitis J: MicroRNA profile analysis of human prostate cancers. Cancer Gene Ther 16: 206-216, 2009.

28. Östling P, Leivonen SK, Aakula A, Kohonen P, Mäkelä R, Hagman Z, Edsjö A, Kangaspeska S, Edgren H, Nicorici D, et al: Systematic analysis of microRNAs targeting the androgen receptor in prostate cancer cells. Cancer Res 71: 1956-1967, 2011.

29. Scapoli L, Palmieri A, Lo Muzio L, Pezzetti F, Rubini C, Girardi A, Farinella F, Mazzotta M and Carinci F: MicroRNA expression profiling of oral carcinoma identifies new markers of tumour progression. Int J Immunopathol Pharmacol 23: 1229-1234, 2010

30. Kanaan Z, Rai SN, Eichenberger MR, Roberts H, Keskey B, Pan J and Galandiuk S: Plasma miR-21: A potential diagnostic marker of colorectal cancer. Ann Surg 256: 544-551, 2012.

31. Gaedcke J, Grade M, Camps J, Søkilde R, Kaczkowski B, Schetter AJ, Difilippantonio MJ, Harris CC, Ghadimi BM, Møller S, et al: The rectal cancer microRNAome-microRNA expression in rectal cancer and matched normal mucosa. Clin Cancer Res 18: 4919-4930, 2012.

32. Xu XM, Qian JC, Deng ZL, Cai Z, Tang T, Wang P, Zhang KH and Cai JP: Expression of miR-21, miR-31, miR-96 and miR-135b is correlated with the clinical parameters of colorectal cancer. Oncol Lett 4: 339-345, 2012.

33. Ma L and Weinberg RA: Micromanagers of malignancy: Role of microRNAs inregulating metastasis. Trends Genet 24: 448-456, 2008.

34. Khew-Goodall Y and Goodall GJ: Myc-modulated miR-9 makes more metastases. Nat Cell Biol 12: 209-211, 2010. 
35. Pang Y, Young CY and Yuan H: MicroRNAs and prostate cancer. Acta Biochim Biophys Sin (Shanghai) 42: 363-369, 2010.

36. Lin SL, Chiang A, Chang D and Ying SY: Loss of mir-146a function in hormone-refractory prostate cancer. RNA 14 417-424, 2008

37. Calò V, Migliavacca M, Bazan V, Macaluso M, Buscemi M, Gebbia N and Russo A: STAT proteins: From normal control of cellular events to tumorigenesis. J Cell Physiol 197: 157-168, 2003.

38. Schindler C and Darnell JE Jr: Transcriptional responses to polypeptide ligands: The JAK-STAT pathway. Annu Rev Biochem 64: 621-651, 1995.

39. Darnell JE Jr: STATs and gene regulation. Science 277: 1630-1635, 1997.

40. Das S, Roth CP, Wasson LM and Vishwanatha JK: Signal transducer and activator of transcription-6 (STAT6) is a constitutively expressed survival factor in human prostate cancer. Prostate 67: 1550-1564, 2007.
41. Fukuda K, Fujitsu Y, Kumagai N and Nishida T: Characterization of the interleukin-4 receptor complex in human corneal fibroblasts. Invest Ophthalmol Vis Sci 43: 183-188, 2002.

42. Ostrand-Rosenberg S, Grusby MJ and Clements VK: Cutting edge: STAT6-deficient mice have enhanced tumour immunity to primary and metastatic mammary carcinoma. J Immunol 165 : 6015-6019, 2000.

43. Ostrand-Rosenberg S, Sinha P, Clements V, Dissanayake SI, Miller S, Davis C and Danna E: Signal transducer and activator of transcription 6 (Stat6) and CD1: Inhibitors of immunosurveillance against primary tumours and metastatic disease. Cancer Immunol Immunother 53: 86-91, 2004. 